\title{
Non-destructive characterisation of all-polypropylene composites using small angle X-ray scattering and polarized Raman spectroscopy
}

\author{
Katalin Bocz $^{1 *}$, Kata Enikő Decsov ${ }^{1}$, Attila Farkas ${ }^{1}$, Dániel Vadas ${ }^{1}$, Tamás \\ Bárány $^{2}$, András Wacha $^{3}$, Attila Bóta ${ }^{3}$, György Marosi ${ }^{1}$ \\ ${ }^{1}$ Department of Organic Chemistry and Technology, Budapest University of \\ Technology and Economics, Müegyetem rkp. 3., H-1111 Budapest, Hungary \\ ${ }^{2}$ Department of Polymer Engineering, Budapest University of Technology and \\ Economics, Mủegyetem rkp. 3., H-1111 Budapest, Hungary \\ ${ }^{3}$ Institute of Materials and Environmental Chemistry, Hungarian Academy of Sciences, \\ Magyar Tudósok krt 2., H-1117 Budapest, Hungary
}

\begin{abstract}
Small angle X-ray scattering (SAXS) and polarized Raman spectroscopy were used to examine the structure of unidirectional all-polypropylene composites prepared at different consolidation temperatures. Analysis of the anisotropy of the X-ray scattering pattern provided a way to quantify the disorientation of the crystallites and a direct correlation has been found between a measure of overall orientation and the Young's modulus of the composites. In the case of the Raman spectroscopic measurements, the molecular orientation state of the reinforcing PP fibres were evaluated by classical least squares (CLS) modelling with real reference spectra. Strong correlation was evinced between the estimated relative degree of orientation of the reinforcing fibres and the Young's modulus of the multi-layered all-polypropylene composites. Based on these

\footnotetext{
*Corresponding author: tel: +36 1-463-1348, e-mail: kbocz@mail.bme.hu, address: Budafoki út 8, Budapest,
} 1111, Hungary
\end{abstract}


results, both SAXS and Raman spectroscopy are suitable methods to predict the mechanical performance of all-polymer composites, being especially sensitive to manufacturing and application conditions, in a non-destructive way.

Keywords: A. Polymer fibres; A. Polymer-matrix composites (PMCs); B. Mechanical properties; D. Non-destructive testing

\section{Introduction}

In self-reinforced polymers (SRPs), the reinforcement and matrix are composed of the same polymer or polymers belonging to the same polymer family. The intensive stretching of extruded tapes or fibres results in high degree of molecular orientation of the polymer chains (both in crystalline and amorphous phase) and by this means polymer fibres of high strength and modulus can be manufactured. The highperformance polymer fibres can be embedded in a matrix polymer by film-stacking [1] or co-extrusion technologies [2,3] serving as suitable reinforcing substances and creating an SRP composite material with low density, enhanced strength, stiffness and impact resistance compared to the unreinforced polymer [4]. By selective melting via hot-compaction [5] or partial dissolution of the skins [6] of the polymer fibres to form the matrix phase, so called single-polymer composites can be created [7]. During production of self-reinforced composites, there are various parameters influencing the properties of the finally formed composite. Besides the ratio and characteristics of the constituents (matrix polymer and reinforcement), the most crucial parameter is the achieved consolidation quality. Full compaction means the least void content and the theoretical maximum density is achieved. The most relevant parameters affecting the consolidation quality are the type and viscosity of the matrix, the type and structure (fibrous, knitted, woven, etc.) of the reinforcement, the volume fraction of the reinforcement, the temperature, pressure and holding-time applied during hot-pressing 
and the cooling speed. It has to be noted, that in contrast to conventional glass or carbon fibre reinforced thermoplastic composites (the consolidation quality of which can be improved by increasing the processing temperature and thus decreasing the viscosity of the matrix), in the case of SRCs the melting temperature of the reinforcing polymer fibre or tape determines the upper limit of the applicable temperature. Therefore, the basis of all SRP techniques is to set a suitable processing window which enables the partial fusing of the fibres (hot-compaction technique) or exploits the difference between the melting temperatures $\left(\mathrm{T}_{\mathrm{m}}\right)$ of the structurally similar reinforcement and matrix polymer. Nevertheless, the increased processing temperature leads to molecular relaxation of fibres and consequently to loss in reinforcing efficiency [8]. In case of allpoly(ethylene terephthalate) (all-PET) composites increased processing temperature and holding time have found to cause hydrolytic degradation, embrittlement and noticeable deterioration of the mechanical properties of the polyester components $[9,10]$. The effect of processing parameters on the mechanical properties was first presented by Ward and Hine for polyethylene (PE) based composites [11] and by Alcock et al. for all-polypropylene composites (all-PPCs) [12,13]. These investigations show that the consolidation quality of all-PPCs improves as a function of increasing processing temperature, as also revealed by tensile, flexural and peel strength, modulus and density measurements by Izer et al. [14]. The modulus and strength values pass through a maximum, while the energy absorption capability (perforation energy) decreases with increasing consolidation temperature. Therefore, it is essential to find the optimal processing conditions, where the SRCs are well-consolidated and accompanied with excellent interlaminar strength (preferably in a presence of transcrystalline layer), and have prominent mechanical performance with good energy absorption at the same time. The optimization and monitoring of the processing parameters are crucial in respect to 
the SRPs quality $[3,10,15,16]$, nevertheless, for the quality assurance of SRP prefabricates or parts during service only destructive or semi-destructive tests are currently available. Quality of the products was mainly assessed by different kind of mechanical loading coupled with further in situ (such as acoustic emission [17]) or post mortem (e.g. light and scanning electron microscopy) failure inspections so far. Nondestructive techniques were rarely used for quality management, though ultrasonic testing [18] and X-ray microcomputed tomography [19] as pioneer investigations can be found in the literature. This is still an open issue, especially if in-line quality inspection is the target [4].

In this work, multi-layered unidirectional all-PPCs, composed of highly stretched isotactic polypropylene (iPP) multifilament fibres and propylene-ethylene random copolymer (rPP) matrix, were investigated. The tensile modulus of the reinforcing iPP fibres basically depends on the fraction of chain segments that are oriented (both amorphous and crystalline orientation) and the degree of orientation along the load direction. From combined Wide Angle X-ray Scattering (WAXS) and Small Angle Xray Scattering (SAXS) experiments the crystallinity, the type and amount of crystals and their lamellar thickness, among others, can be obtained [20,21]. However, for estimation of the mechanical properties of multi-layered all-PPCs, prepared at varying composite processing temperatures, the investigation of the thermally induced relaxation and structural transitions of the iPP fibres needed a simplified approach in the data evaluation of the X-ray patterns. The overall anisotropy was planned to be quantified by means of azimuthal scattering curves.

Beside scattering methods, Polarized Raman spectroscopy proved to be effective to study regularity and structure of iPP fibres $[22,23,24]$. In the former studies, the ratio of Raman bands at $808 \mathrm{~cm}^{-1}$, representing the helical conformation of the chains present 
in crystalline region, and $841 \mathrm{~cm}^{-1}$, representing the defects in the helix, was generally used to estimate the overall molecular orientation (including crystalline and amorphous phases) $[23,25,26]$. However, the structure of reinforcing iPP fibres embedded in a structurally similar polymer (rPP) matrix has not been investigated yet. For this purpose, the entire frequency range of the Raman spectra, collected from all-PPCs, was proposed to be evaluated by a multivariate evaluation method [27]. In this work, the classical least squares (CLS) model was selected for determining the relative macromolecular orientation of the reinforcing fibres and thus estimating their mechanical performance at the same time.

\section{Materials and methods}

\subsection{Materials}

Highly oriented isotactic polypropylene (iPP) homopolymer multifilament (Stradom S.A., Poland) was used as reinforcement. The reinforcing multifilament has a melting temperature of $171^{\circ} \mathrm{C}$, as determined by DSC from the first melting curve with a heating rate of $5^{\circ} \mathrm{C} / \mathrm{min}$ (shown in Figure 1), single fibre diameter of $34.4 \pm 1.1 \mu \mathrm{m}$, tensile strength of $581 \pm 30 \mathrm{MPa}$ and tensile modulus of $7687 \pm 932 \mathrm{MPa}$ (determined between strain levels of 0.1 and $0.3 \%$ ), as measured in single fibre tensile tests.

A propylene-ethylene copolymer (rPP) based thermoplastic elastomer (Versify 4200, Dow Chemical Company, USA) was selected as matrix material. According to DSC measurements with a heating rate of $5^{\circ} \mathrm{C} / \mathrm{min}$, the rPP has a broad crystalline melting temperature range between 55 and $100^{\circ} \mathrm{C}$ with a peak temperature of $78^{\circ} \mathrm{C}$ (as can be seen from Figure 1). Accordingly, the processing window, approximated by the 
difference between the melting temperatures of the reinforcement and matrix material, is about $70^{\circ} \mathrm{C}$.

$60 \pm 5 \mu \mathrm{m}$ thick films were manufactured from the rPP granules by film extrusion technique using a Labtech LCR 300 laboratory flat film line (Labtech Engineering Co., Thailand).

\section{Figure 1 DSC curves of the iPP fibre and rPP matrix}

\subsection{Composite preparation}

All-PPC sheets were prepared by filament winding followed by compression moulding process [28]. 5 plies of matrix films and 4 iPP multifilament layers were laminated tightly onto a $6 \mathrm{~mm}$ thick aluminium core $(300 \times 300 \mathrm{~mm})$ in a filament winding process to obtain unidirectionally (UD) aligned fibres. The all-PPCs with a thickness of $1.7 \mathrm{~mm}$ and a nominal reinforcing multifilament content of $65 \mathrm{wt} \%$ were produced by compression moulding. The filament-wound laminates were consolidated at different temperatures; at $130,140,150,160,170$ and $180^{\circ} \mathrm{C}$, respectively, serving as series regarding molecular orientation of fibres and mechanical performance. The filmstacked packages were inserted in between the preheated moulds and held for $300 \mathrm{~s}$ without pressure, then compressed for $420 \mathrm{~s}$ under a pressure of $1 \mathrm{MPa}$ and finally cooled to $45^{\circ} \mathrm{C}$ (under pressure).

\subsection{Methods}

\subsubsection{Tensile tests}

Comparative static tensile tests were performed on water jet cut rectangular composite specimens of $20 \mathrm{~mm} \times 120 \mathrm{~mm}$ (width $\times$ length) (the gauge length was 70 
mm) using a Zwick Z020 universal testing machine (Zwick GmbH \& Co. KG, Germany) with a crosshead speed of $5 \mathrm{~mm} / \mathrm{min}$. Young's modulus values were determined from the stress-strain curves between the strain levels of 0.1 and $0.5 \%$ (the specimen strain was measured by using a Messphysik ME 46 type (Messphysic, Austria) video extensometer).

\subsubsection{SAXS}

Small-angle X-ray scattering measurements were carried out on the CREDO instrument [29] of the Research Centre for Natural Sciences, Hungarian Academy of Sciences (Budapest, Hungary). The sample platelets were mounted on the sample holder without further treatment, the direction of the fibres being nearly vertical, as shown in

Figure 2. X-rays were produced by a $\mathrm{GeniX}^{3 \mathrm{D}} \mathrm{Cu}$ ULD integrated beam delivery system (Xenocs SA, Sassenage, France), which consists of a microfocus $\mathrm{Cu}$ anode $\mathrm{X}$ ray tube (30 W nominal power, $30 \mu \mathrm{m}$ thermal focus) operated at $50 \mathrm{kV}$ and $0.6 \mathrm{~mA}$ and a FOX3D multilayer parabolic mirror. The divergence and the diameter of the $\mathrm{X}$ ray beam were limited by a 3-pinhole collimation scheme, resulting in a highly parallel beam of X-rays with a cca. $1 \mathrm{~mm}$ diameter [30]. Scattered photons were recorded using a Pilatus-300k CMOS hybrid pixel detector (Dectris Ltd, Baden, Switzerland), placed $2415.8 \mathrm{~mm}$ downstream from the sample. This determined the resolved range of the momentum transfer ( $q=4 \pi \sin \theta / \lambda$, where $2 \theta$ is the scattering angle and $\lambda=0.154$ $\mathrm{nm}$ is the X-ray wavelength of $\mathrm{Cu} \mathrm{K \alpha}$ radiation) between 0.034 to $1.168 \mathrm{~nm}^{-1}$, corresponding to 182.5 to $5.4 \mathrm{~nm}$ Bragg distances. To minimize the effects of sample inhomogeneity, scattering images were recorded at 15 positions across the breadth of the sample, placed $0.8 \mathrm{~mm}$ apart (the diameter of the circular X-ray beam at the sample was cca. $1 \mathrm{~mm}$ ). At each position, 12 exposures were made, each 120 second long. 
Frequent re-measuring of the calibrant samples and instrumental background was also included in the procedure. All scattering patterns were corrected for instrumental background, sample self-absorption and geometrical distortions and normalized into absolute intensity units by the standard data reduction algorithm implemented in the instrument control software. The abscissa was calibrated from pixel edge length units into the scattering variable $(q)$ using a pre-calibrated specimen of silver behenate. For the intensity calibration, a piece of glassy carbon with known scattering cross-section has been used.

The distinct exposures were averaged for each sample and the ones affected by external radiation background have been excluded using statistical tests. Finally, the complete scattering images for each sample were radially averaged in the range of $0.04 \leq q \leq 0.1 \mathrm{~nm}^{-1}$ to obtain azimuthal scattering curves, i.e. the intensity as a function of the azimuth angle of the scattering pattern.

\section{Figure 2 Schematic representation of the experimental setup for small-angle $X$-ray}

\section{scattering}

\subsubsection{Polarized Raman micro-spectroscopy}

Raman spectra were collected using a Horiba Jobin-Yvon LabRAM system coupled with an external $785 \mathrm{~nm}$ diode laser source and an Olympus BX-40 optical microscope. An objective of 50x magnification was used for optical imaging and spectrum acquisition. The laser beam is directed through the objective, and backscattered radiation is collected with the same objective. The collected radiation is directed through an edge filter that removes the Rayleigh photons, then it passes through a confocal hole and the entrance slit and gets onto a grating monochromator 
(950 groove $\mathrm{mm}^{-1}$ ) that disperses the light before it reaches the CCD detector. The spectrograph was set to provide a spectral range of $200-1400 \mathrm{~cm}^{-1}$ with a $2 \mathrm{~cm}^{-1}$ resolution.

The schematic representation of the experimental setup for Raman imaging is shown in Figure 3. The incident laser radiation propagates in the negative $\mathrm{Z}$ direction with polarization in $\mathrm{Y}$ direction. When a half-wave plate $(\lambda / 2)$ was inserted, the polarization direction of the linearly polarized light shifted to $\mathrm{X}$ direction. The UD composite samples were mounted horizontally with reinforcing fibres oriented in the $\mathrm{Y}$ direction in all cases, so thus the electric field of excitation was parallel (Y) or perpendicular $(\mathrm{X})$ to the fibre orientation.

\section{Figure 3 Schematic representation of the experimental setup for Raman imaging}

Reference Raman spectra were collected from the matrix material (rPP) (test specimen was fabricated from a $2 \mathrm{~mm}$ thick plate obtained after hot-pressing the rPP granules at $160^{\circ} \mathrm{C}$ ) and from the reinforcing iPP fibre in its original highly oriented form ( $\mathrm{iPP}_{\mathrm{o}-100 \%}$ ), and in its completely heat relaxed form ( $\left.\mathrm{iPP}_{\mathrm{o}-0 \%}\right)$ obtained after heat treating (melting) $5 \mathrm{~g}$ of iPP fibre at $180^{\circ} \mathrm{C}$ for 20 minutes in an oven (and let them cool without pressure). The Raman spectra of the references were collected with a $100 x$ objective using an acquisition time of $90 \mathrm{~s}$ and averaging 3 measured spectra at each measured point.

Raman spectra were collected along the depth of the composites to create line maps and to determine the optimal depth for two-dimension (2D) Raman mapping. Small area maps were collected with 50x objective (laser spot size: $\sim 2.0 \mu \mathrm{m}$ ) and $6 \mu \mathrm{m}$ and $12 \mu \mathrm{m}$ step size in the $\mathrm{X}$ (perpendicular to the fibre orientation) and in the $\mathrm{Y}$ 
direction (parallel to the fibre orientation), respectively. In each experiment the acquisition time of a single spectrum was $30 \mathrm{~s}$ and 3 such spectra were averaged at each pixel. The analysed area was $36 \times 12$ pixels, i.e. $210 \times 132 \mu^{2}$ in all cases.

\section{Results and discussion}

\subsection{Tensile tests}

The tensile strength and modulus values determined for the examined all-PPCs prepared at different consolidation temperatures are summarized in Table 1. These data were used as reference for comparison of the estimated molecular alignment of the composites and reinforcing fibres by SAXS and Raman spectrometry, respectively. The Young's modulus of the composites was found to be more sensitive to the increasing processing temperatures. It can be seen that the tensile strength of the all-PPC consolidated at $170^{\circ} \mathrm{C}$, at the melting temperature of the used iPP fibre, is still approximately the same as that of the all-PPCs prepared at lower temperatures, but its tensile modulus already decreases by $45 \%$ compared to the all-PPC prepared at $130^{\circ} \mathrm{C}$. The drop in tensile modulus while tensile strength remains constant can be explained by the fact that modulus is more dependent on the orientation of the amorphous phase, while tensile strength is more dependent on crystalline orientation and structural effects [31]. In agreement with this effect, even though the shrinkage of wound fibres is hindered, microstructural changes such as relaxation and thus noticeable decrease in tensile modulus can occur during processing into all-PPCs without any visible indications [32]. This observation highlights that the precise monitoring and control of the composite preparation parameters is crucial in respect to the product's quality. 
Table 1 Tensile strength and modulus of the all-PPCs prepared at different consolidation temperatures

\subsection{SAXS}

SAXS was used to characterize the anisotropy of the composites on the nanometre scale. The SAXS patterns of the samples, shown in Fig. 4, demonstrate strong dependence on the thermal prehistory and definite changes in the orientation. The most striking part is the high-intensity horizontal streak, which is a direct result of vertically extended scattering objects, typically the nanocrystallites in the iPP fibres. With increasing consolidation temperature these streaks decrease in intensity even below the melting point, but once this temperature is surpassed, they vanish completely, giving place of a new scattering signal in the vertical direction, which can be attributed to broken remnants of the original crystallites and isotropically recrystallized domains.

\section{Figure 4 Small-angle X-ray scattering patterns of the all-PPCs prepared at different consolidation temperatures}

The overall anisotropy can be quantified by means of azimuthal scattering curves. As the most significant changes to the anisotropy occur at the very small $q$-range, we have chosen the $0.05-0.1 \mathrm{~nm}^{-1}$ interval (a thin ring very near the centre of the image, just apart from the beam stop) to calculate the dependence of the intensity on the azimuth angle of the scattering pattern. The scattering curves are shown in Fig. 5.

Figure 5 Azimuthal scattering curves of the all-PPCs in the range of $0.05-0.1 \mathbf{n m}^{-1}$ 
As seen from the figure, the main peak pair (cca. at $90^{\circ}$ and $270^{\circ}$ ) corresponds to the horizontal streaks. Additionally, another peak pair emerges at higher temperatures orthogonally to the previous two, at $0^{\circ}$ and $180^{\circ}$ (and of course $360^{\circ}$ ), as a contribution of the recrystallized iPP fibrils. To obtain a quantitative characteristic of the nanocrystalline system, the azimuthal curves were fitted in the least-squares sense with a model of two sets of Gaussian peaks, placed $180^{\circ}$ apart. The peaks in each set had common height and width parameters. Additionally, a constant baseline term was used to model the isotropic scattering.

The width of the horizontal streaks can be regarded as a measure of the orientation of the lamellas: the narrower the streaks, the better the crystallites are oriented. It can be expected that with the loss of orientation of the reinforcing iPP crystallites the stiffness of the material in that direction should also decrease. The strong correlation (coefficient of determination $\mathrm{R}^{2}=0.983$ ) between the Young's moduli and the reciprocal of the width parameter of the peaks at $90^{\circ}$ and $270^{\circ}$ shown in Fig. 6 fulfils this expectation nicely. For closer examination of the changes of crystalline and amorphous orientation and to understand their contribution to the evolution of the macroscopic properties during processing, comparison of SAXS and WAXS measurements is planned.

Figure 6 Correlation of Young's moduli with the reciprocal width of the horizontal streak 


\subsection{Raman spectroscopy}

\subsubsection{Depth profiling of orientation ratio by line mapping}

Orientation ratio (OR) along line maps was determined as proposed by Chen et al. as a simple and robust method for characterisation of the molecular orientation across the cross-section of extruded oriented polymer composite samples using polarized micro-Raman spectroscopy [33]. In Equation (1) $I_{808}$ and $I_{841}$ stand for the intensity of Raman bands at 808 and $841 \mathrm{~cm}^{-1}$, respectively. The $X$ and $Y$ in subscript denote whether the spectrum is collected with the electric field of the applied excitation beam is parallel or perpendicular to the direction of the iPP fibres.

$$
O R=\frac{I_{808, X} / I_{841, X}}{I_{808, Y} / I_{841, Y}}
$$

The OR for the all-PPC constituents was determined based on the spectra shown in Figure 7, and was calculated to be 9.3 for the iPP fibre and 1.4 for the hot-pressed rPP matrix material, respectively. The OR of 9.3 is a high value indicating very high draw ratio of the fibres, while for a completely relaxed sample the OR should be close to 1 as the polarization should not affect Raman spectra of isotropic materials.

\section{Figure $7 \mathrm{Y}$ and $\mathrm{X}$ spectra of the $\mathrm{PPP}$ matrix and the iPP fibre}

Two line maps with differing polarisation directions ( $\mathrm{Y}$ and $\mathrm{X}$ ) were collected from the same, evenly spaced points from the surface into the core from each all-PPC sample. The obtained OR profiles, representing the changes in the overall orientation (including crystalline and amorphous phases), are shown in Figure 8. It can be seen that 
the OR values increase with depth in all cases; in the first $50 \mu \mathrm{m}$ radiation is mainly collected from the isotropic matrix material with low OR, then as we proceed deeper more and more photons are backscattered from the high-tenacity iPP fibres causing increase of the OR, while around $100 \mu \mathrm{m}$ depth the OR reaches a plateau indicating that the majority of the photons are collected from the iPP fibres. It can also be observed that the maximal OR values calculated for the all-PPC samples decrease with increasing processing temperatures. A maximal OR of 9.1, close to that of the neat iPP fibre (9.3), was determined for the composite prepared at $130^{\circ} \mathrm{C}$ indicating that at this processing temperature the reinforcing iPP fibre could maintain its highly ordered structure. In contrast, based on the noticeable lower maximal OR value of the all-PPC prepared at $180^{\circ} \mathrm{C}$, considerable structural changes (relaxation) of the reinforcing fibre were revealed.

\section{Figure 8 OR profiles collected from the surface region of the all-PPCs}

In Figure 9, fairly good correlation (Pearson's $r=0.884$ ) can be observed between the determined maximal OR values and the tensile moduli of the all-PPCs. The better the correlation the more accurate could be the Raman spectroscopy based indirect

estimation of the mechanical performance of the all-PPCs. In order to improve the estimation accuracy, small-area maps, being more representative regarding the orientation state of the reinforcement, were analysed.

Figure 9 Correlation of Young's moduli with the maximal OR determined for the all-PPCs 


\subsubsection{CLS modelling of small-area maps}

Raman mapping contributes to further understanding of the orientation and relaxation process through visualization of the structural changes (including overall molecular orientation and crystallinity) in all-PPCs. Based on the OR profiles given by the line maps in Figure 8, the Raman maps were decided to be collected from $125 \mu \mathrm{m}$ depth from the surface of the composites. At this depth the intensity of the Raman scattering was still sufficient (much deeper sampling would have caused significant intensity loss). Furthermore, the multivariate evaluation of the Raman spectra was expected to increase the accuracy of the estimation of the morphological changes, including the degree of crystallinity and extent of overall macromolecular orientation, of the reinforcing iPP fibres.

Reference Raman spectra were collected with excitation polarization in the $\mathrm{Y}$ direction from the highly oriented iPP fibre $\left(\mathrm{iPP}_{\mathrm{o}-100 \%}\right)$, from the fully relaxed iPP fibre $\left(\mathrm{iPP} \mathrm{o}_{\mathrm{o}-\%}\right)$ and from the matrix polymer (rPP) (Figure 10). The spectra collected from iPP fibre both in oriented and relaxed form match the typical Raman shifts of a semicrystalline iPP, the vibrational assignments are given in Table 2 [34]. The noticeable differences in the peak intensities and ratios of the highly oriented (iPP $\mathrm{P}_{0-100 \%}$ ) and relaxed iPP fibres ( $\mathrm{iPP}_{\mathrm{o}-0 \%}$ ) provide a good basis for estimating the structure formation of the reinforcing fibres during composite processing including heat and compression exposure. In contrast, the rPP matrix shows great similarity to the spectrum of the heat relaxed iPP fibre (iPP $\mathrm{i}_{\mathrm{-}-0 \%}$ ), which makes the modelling and estimation fairly difficult. As for a solution, instead of evaluating only the Raman peak intensity ratios at $808 \mathrm{~cm}^{-1}$ and $841 \mathrm{~cm}^{-1}$, as suggested by Paradkar et al. [25], CLS method was applied to the entire frequency range $\left(200-1400 \mathrm{~cm}^{-1}\right)$, providing better estimation for the orientation state of the iPP fibres embedded in rPP matrix. 
Before chemometric evaluation, all spectra were baseline corrected and normalized in order to eliminate the intensity deviation between the measured points. The morphological changes (overall molecular orientation and crystallinity) of the reinforcing iPP fibres of the all-PPCs as a function of increasing consolidation temperature were evaluated in relation to the initial morphology of the reference constituents. The relative orientation state was determined by CLS modelling with the real reference spectra collected from $\mathrm{iPP}_{0-100 \%}, \mathrm{iPP}_{\mathrm{o}-0 \%}$ and $\mathrm{rPP}$. Each Raman spectrum obtained from an all-PP composite is assumed as a linear combination of the three reference spectra. All changes in the spectra are equally weighted. The relative degree of orientation of the iPP fibre is estimated after subtracting the contribution of the matrix component. It is defined regarding its relation to the reference spectra of $\mathrm{iPP}_{\mathrm{o}}$ $100 \%$, being considered as $100 \%$ relative orientation state, and to $\mathrm{iPP}_{\mathrm{o}-0 \%}$ as $0 \%$ relative orientation state.

Figure 10 Reference Raman spectra collected from iPP $\mathbf{p}_{0-100 \%}, \mathbf{i P P}_{0-0 \%}$ and $\mathbf{r P P}$

Table 2 Vibrational assignments for Raman bands of iPP [34]

Raman maps were collected from $0.02772 \mathrm{~mm}^{2}$ area of each all-PPC prepared at consolidation temperatures between $130^{\circ} \mathrm{C}$ and $180^{\circ} \mathrm{C}$ and analysed using CLS method. The maps of $\mathrm{iPP}_{\mathrm{0}-100 \%}$ contribution calculated for the all-PPCs consolidated at different temperatures are shown in Figure 11. With increasing processing temperature the area of darker (blue or red) domains corresponding to the less ordered structures clearly increases. Below the starting temperature of the crystalline melt $\left(158.5^{\circ} \mathrm{C}\right)$ only slight relaxation occur during heat treatment, while above $160^{\circ} \mathrm{C}$ the increasing mobility of the 
polymer chains, trying to return to the thermodynamically stable coil state, results in noticeably decreased orientation degrees. Based on the uneven interfaces and smaller characteristic orientation degrees considerable molecular relaxation and infusion of fibre/matrix interfaces were evinced for the fibres embedded in the all-PPC prepared at $170^{\circ} \mathrm{C}$ and $180^{\circ} \mathrm{C}$

Figure 11 Maps of $\operatorname{iPP}_{\mathbf{0}-100 \%}$ contribution estimated for the all-PPCs

The average relative degrees of orientation obtained from CLS modelling the smallarea Raman maps of the examined all-PPCs are shown in Figure 12 in relation to the composites' Young's moduli. Fairly strong correlation (Pearson's $r=0.915$ ) was evinced between the tensile moduli and the relative orientation degrees (estimated based on the polarized Raman maps of the all-PPCs), indicating improved adequacy of multivariate evaluation of small-area maps compared to the determination of orientation ratios of line maps. It can be noted, however, that Raman spectroscopy is sensitive to the overall chain conformation but insensitive to the lateral order of the crystalline phase, unlike SAXS technique. Furthermore, during CLS modelling all changes in the spectra are equally weighted, but the macroscopic properties like Young’s modulus may be more influenced by the variation of specific factors such as orientation of the amorphous region.

In this work, the Raman spectra were acquired at an accumulation time of $30 \mathrm{~s}$, but in situ results of Martin et al. [35] evidenced the ability of the Raman method to characterize molecular orientation also in real-time with less than $5 \mathrm{~s}$ time resolution. Transmission Raman spectroscopy may also offer rapid and even better sampling for solids than conventional backscatter Raman. It is presumed that Raman spectroscopy 
based techniques can serve as core units of an on-line control loop aiming at the precise control of the manufacturing process (such as continuous double-belt pressing) of selfreinforced composites, being especially sensitive to the preparation parameters (such as consolidation temperature, time, pressure, etc.).

\section{Figure 12 Correlation of Young's moduli with the estimated relative degree of orientation values in all-PPC samples}

\section{Conclusions}

Both small-angle X-ray scattering and polarized Raman spectroscopy proved to be suitable methods to characterize the structure of reinforcing iPP fibres of all-PP composites in a non-destructive way.

Small-angle X-ray scattering measurements helped to evince the changes of orientation in the iPP fibres on the nanometric level. Analysis of the anisotropy of the scattering pattern provided a way to quantify the disorientation of crystallites and a direct correlation has been found between a measure of orientation and the Young's moduli of the composites.

Based on the Raman spectra collected from the all-PPCs, the orientation states of the embedded iPP fibres were determined, as a new approach, by multivariate modelling with real reference spectra. Furthermore, visualization of the morphological changes could be performed through Raman mapping. The strong correlation evinced between the tensile moduli of the all-PPCs and the estimated relative orientation degrees of the iPP fibres served as proof for the adequacy of the elaborated method.

Comparing the two methods, SAXS provides more subtle morphological details and accurate correlation, while the polarized Raman spectroscopy, due to its quick 
sampling and flexibility, is suitable for establishing real time monitoring and Raman signal based process control. Thus it is expected to gain application in self-reinforced composite manufacturing and also in other fields where ordered structure of macromolecules is relevant.

\section{Acknowledgement}

The research was financially supported by the Hungarian Scientific Research Fund (OTKA K112644 and PD121171) and by the Higher Education Excellence Program of the Ministry of Human Capacities in the framework of the Nanotechnology research area of the Budapest University of Technology and Economics (BME FIKP-NANO). This work was supported by the National Research, Development and Innovation Fund in the frame of NVKP_16-1-2016-0012 and GINOP-2.2.1-15-2016-00015 projects. The

CREDO research instrument has been funded jointly by Gedeon Richter Plc, the Hungarian National Scientific Research Fund (grant No. CNK 810520) and the Central Hungarian Operative Program of the National Research, Development and Innovation Fund (KMOP-1.1.2-07/1-2008-0002). K. Bocz is thankful for the János Bolyai Research Scholarship of the Hungarian Academy of Sciences.

\section{References}

[1] Zhang JM, Reynolds CT, Peijs T. All-poly(ethylene terephthalate) composites by film stacking of oriented tapes. Composites Part A 2009;40:1747-1755.

[2] Peijs T. Composites for recyclability. Mater Today 2003;6:30-35. 
[3] Kim KJ, Yu WR, Harrison P. Optimum consolidation of self-reinforced polypropylene composite and its time-dependent deformation behavior. Composites Part A 2008;391597-1605.

[4] Karger-Kocsis J, Bárány T. Single-polymer composites (SPCs): Status and future trends. Compos Sci Technol 2014;92:77-94.

[5] Hine PJ, Ward IM. The hot compaction of woven polypropylene tapes. J Mater Sci 1998;33:2725-2733.

[6] Zhang JM, Mousavi Z, N Soykeabkaew, Smith P, Nishino T, Peijs T. All-aramid composites by partial fiber dissolution. Appl Mater Interfaces 2010;2:919-926.

[7] Alcock B, Peijs T. Technology and Development of Self-Reinforced Polymer Composites. In: Abe ., Kausch HH, Möller M, Pasch H. (eds) Polymer Composites Polyolefin Fractionation - Polymeric Peptidomimetics - Collagens. Advances in Polymer Science, vol 251. Springer, Berlin, Heidelberg, 2011

[8] Bocz K, Simon D, Bárány T, Marosi G. Key role of reinforcing structures in the flame retardant performance of self-reinforced polypropylene composites. Polymers 2016;8:289

[9] Hine P. J., Ward I. M.: Hot compaction of woven poly(ethylene terephthalate) multifilaments. J Appl Polym Sci 2004;91:2223-2233.

[10] Chen JC, Wu CM, Pu FC, Chiu CH. Fabrication and mechanical properties of selfreinforced poly(ethylene terephthalate) composites. Express Polym Lett 2011;5:228237.

[11] Ward IM, Hine PJ. Novel composites by hot compaction of fibers. Polym Eng Sci 1997;37:1809-1814. 
[12] Alcock B, Cabrera NO, Barkoula NM, Peijs T. Low velocity impact performance of recyclable all-polypropylene composites. Comp Sci Technol 2006;66:1724-1737.

[13] Alcock B, Cabrera NO, Barkoula NM, Loos J, Peijs T. The mechanical properties of unidirectional all-polypropylene composites. Composites Part A 2006;37:716-726.

[14] Izer A, Bárány T, Varga J. Development of woven fabric reinforced allpolypropylene composites with beta nucleated homo- and copolymer matrices, Compos Sci Technol 2009;69:2185-92.

[15] Swolfs Y, Zhang Q, Baets J, Verpoest I. The influence of process parameters on the properties of hot compacted self-reinforced polypropylene composites. Composites Part A 2014;65:38-46.

[16] Diaz JA, Youngblood JP. Multivariable dependency of thermal shrinkage in highly aligned polypropylene tapes for self-reinforced polymer composites. Composites Part A 2016;90:771-777

[17] Izer A, Stocchi A, Bárány T, Pettarin V, Bernal C, Czigány T. Effect of the consolidation degree on the fracture and failure behavior of self-reinforced polypropylene composites as assessed by acoustic emission. Polym Eng Sci 2010;50:2106-13.

[18] Heim HP, Tillmann W, Ries A, Sievers N, Rohde B, Zielke R. Visualisation of the degress of compaction of self-reinforced polypropylene composites by means of ultrasonic testing, J Plast Technol 2013;9:275-94.

[19] Kiss Z, Kmetty Á, Bárány T. Investigation of the weldability of the self-reinforced polypropylene composites, Mater Sci Forum 2010;659:25-30. 
[20] Housmans JW, Gahleitner M, Peters GWM, Han E.H. Meijer HEH. Structureproperty relations in molded, nucleated isotactic polypropylene. Polymer 2009;50:2304-2319.

[21] Fischer S, Marti O, Diesner T, Rieger B. Small-angle X-ray scattering on meltspun polypropylene fibers: modeling and data reduction. Macromolecules 2010;43:5009-5015.

[22] Fraser G, Hendra P, Watson D, Gall M, Willis H, Cudby M. The Raman spectra of oriented isotactic polypropylene. Spectrochim Acta A Mol Biomol Spectros 1995;51:2117-2124.

[23] Ran S, Fang D, Sics I, Toki S, Hsiao BS, Chu B. Combined techniques of Raman spectroscopy and synchrotron X-ray for in-situ studies of polypropylene fibers during tensile deformation. Rev Sci Instrum 2003;74:3087-3092.

[24] Kida T, Hiejima Y, Nitta KH. Molecular orientation behavior of isotactic polypropylene under uniaxial stretching by rheo-Raman spectroscopy. Express Polym Lett 2016;10,701-709.

[25] Paradkar RP, Sakhalkar SS, He X, Ellison MS. OnLine estimation of Molecular Orientation in Polypropylene Fibers Using Polarized Raman Spectroscopy. J Appl Spectrosc 2001;55:534-539.

[26] Lee BH, Cho JW, Kim KH. Crystallization, orientation, and mechanical properties of laser-heated photothermally drawn polypropylene/multi-walled carbon nanotube fibers. Eur Polym J 2017;91:70-80.

[27] Bocz K. Development of environmentally friendly flame retarded polymer composites, PhD Thesis, Budapest University of Technology and Economics, 2015 
[28] Kmetty Á, Tábi T, Kovács JG, Bárány T. Development and characterisation of injection moulded, all-polypropylene composites. Express Polym Lett 2013;7:134-145.

[29] Wacha A, Varga Z, Bóta A. CREDO: a new general-purpose laboratory instrument for small-angle X-ray scattering. J Appl Cryst 2014;47:1749-1754.

[30] Wacha A. Optimized pinhole geometry for small-angle scattering. J Appl Cryst 2015;48:1843-1848

[31] Flood JE, Nulf SA. How molecular weight distribution and drawing temperature affect polypropylene physical properties and morphology. Polym Eng Sci 1990;30:1504-1512.

[32] Alcock B, Cabrera NO, Barkoula NM, Peijs T. The effect of processing conditions on the mechanical properties and thermal stability of highly oriented PP tapes Eur Polym J 2009;45:2878-2894.

[33] Chen X, Leugers MA, Kirch T, Stanley J. Orientation Mapping of Extruded Polymeric Composites by Polarized Micro-Raman Spectroscopy. J Spectrosc 2015; Article ID 518054, 7 pages

[34] Nielsen AS, Batchelder DN, Pryz R. Estimation of crystallinity of isotactic polypropylene using Raman spectroscopy. Polymer 2002;43:2671-2676.

[35] Martin J, Poncot M, Bourson P, Dahoun A, Hiver JM. Study of the crystalline phase orientation in uniaxially stretched polypropylene by Raman spectroscopy: validation and use of a time-resolved measurement method. Polym Eng SCi 2001;51:1607-1616. 